\title{
A phase II trial of thymidine and carboplatin for recurrent malignant glioma: A North American Brain Tumor Consortium Study ${ }^{1}$
}

\author{
H. Ian Robins, ${ }^{2}$ Susan M. Chang, Michael D. Prados, W.K. Alfred Yung, Kenneth \\ Hess, David Schiff, Harry Greenberg, Karen Fink, Kelly Nicolas, John G. Kuhn, \\ Timothy Cloughesy, Larry Junck, and Minesh Mehta
}

Department of Medicine, University of Wisconsin, Madison, WI 53792 (H.I.R., M.M.); University of

California at San Francisco, San Francisco, CA 94143 (S.M.C., M.D.P., K.N.); University of Texas MD

Anderson Cancer Center, Houston, TX 77030 (W.K.A.Y., K.H.); University of Pittsburgh, Pittsburgh, PA 15213

(D.S.); Department of Neurology, University of Michigan, MI 48109 (L.J., H.G.); University of Texas

Southwestern at Dallas, Dallas, TX 75390 (K.F.); University of Texas at San Antonio, San Antonio, TX 78284

(J.G.K.); University of California at Los Angeles, Los Angeles, CA 90095 (T.C.)

This phase II study in recurrent high-grade glioma evaluated the response rate, toxicities, and time to treatment failure of high-dose carboplatin modulated by a $24-\mathrm{h}$ infusion of thymidine $\left(75 \mathrm{~g} / \mathrm{m}^{2}\right)$. The trial was based on preclinical data and a prior phase I study (J. Clin. Oncol. 17, 2922-2931, 1999); a phase II recurrent high-grade glioma study was initiated in July of 1998 . Thymidine was given over $24 \mathrm{~h}$; carboplatin was given over $20 \mathrm{~min}$ at hour 20 of the thymidine infusion. The starting dose of carboplatin had a value of 7 for the area under the curve (AUC), with allowance for dose escalation of 1 AUC unit per cycle if grade 2 toxicity was observed. Treatment cycles were repeated every 4 weeks. Accrual as of September 1999 was 45 patients [ 4 were unevaluable]: $76 \%$

Received 10 September 2001, accepted 26 October 2001.

${ }^{1}$ Supported by a 2001 grant (CA62399 [CA62422, CA62421, CA62426, CA62405-07, CA62455, CA62407, CA62455-08]) and an MO1 (General Clinical Research Center) grant (RR03186-11, RR00056, RR00633, RR00079, RR00042, RR0865) from the National Institutes of Health; grants from the Kathleen Reader Neuro-Oncology Research Fund; and the Teams Research Fund.

${ }^{2}$ Address correspondence and reprint requests to $\mathrm{H}$. Ian Robins, Department of Medicine, University of Wisconsin Comprehensive Cancer Center K4/666, 600 Highland Ave., Madison, WI 53792.

${ }^{3} \mathrm{Abbreviations}$ used are as follows: AUC, area under the curve; $\mathrm{Cl}$, confidence interval; GBM, glioblastoma multiforme. with glioblastoma multiforme (GBM), $20 \%$ with anaplastic oligodendroglioma, $2 \%$ with mixed type, and $2 \%$ with anaplastic astrocytoma. Most patients had prior chemotherapy $(78 \%)$. As observed in the earlier phase I study (in which carboplatin pharmacokinetics were unaltered by thymidine or antiseizure medications), thymidine was myeloprotective, resulting in a minimal need for dose reduction for patients having a $>2$ grade toxicity (in only $4 \%$ of the courses of treatment). Of 101 total courses, the number of courses (at the AUCs) was 3 (5), 4 (6), 58 (7), 20 (8), 11 (9), and 5 (10). Grade 3 nonhematologic toxicities included headache $(4 \%)$, altered consciousness $(3 \%)$, fatigue $(1 \%)$, and nausea $(3 \%)$. Responses included 2 partial ( 1 oligodendroglioma, 1 GBM; 5\%); 3 minor (1 anaplastic astrocytoma, 2 GBM; $7.3 \%)$; 6 stable disease $(14.6 \%)$; and 30 progressive disease $(73.2 \%)$. For GBM patients, median survival was 23 weeks (with a $95 \%$ confidence interval of 20 to 50 weeks), and progression-free survival was 8 weeks (with a $95 \%$ confidence interval of 7-16 weeks). These results in GBM were comparable to other phase II GBM trials and thus do not represent a therapeutic advance in the treatment of GBM. Taken collectively, however, results are consistent with continued investigation of thymidine in combination with chemotherapeutic agents for high-grade glioma and other malignant diseases. The significant myeloprotection afforded by thymidine may have particular relevance to polychemotherapeutic regi- 
mens. Neuro-Oncology 4, 109-114, 2002 (Posted to Neuro-Oncology [serial online], Doc. 01-051, February 1, 2002. URL <neuro-oncology.mc.duke.edu>)

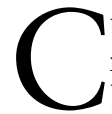
urrently, the overall survival of recurrent malignant glioma is in the order of 4 to 6 months (Kornblith and Walker, 1988). Most salvage therapies produce limited benefit without a clear survival advantage. Standard salvage therapies include various chemotherapeutic agents, repeat resection, and radiotherapeutic interventions such as radiosurgery or interstitial implantation.

Thymidine is a naturally occurring nucleoside that preferentially kills neoplastic cells in vitro and induces regression in a wide variety of tumor xenografts including malignant gliomas (Cohen et al., 1989, 1990). Prolonged infusions of thymidine produce responses in heavily pretreated patients, but are associated with considerable myelotoxicity (Kufe et al., 1980a, 1980b; O'Dwyer et al., 1987). Shorter infusions (e.g., 24 h) maintain antineoplastic activity preclinically and are free of myelosuppression (Cohen et al., 1989, 1990).

Mechanistically, thymidine undergoes intracellular triphosphorylation. The triphosphate modulates ribonucleotide reductase, resulting in deoxycytidine starvation, an increase in deoxyguanosine triphosphate, and inhibition of poly(ADP-ribose) polymerase (a key repair enzyme), which results in cessation of DNA synthesis, inhibition of repair of DNA damage, and cell death (O'Dwyer et al., 1987). Inhibition of poly(ADP-ribose) polymerase allows considerable enhancement (2.5- to 3 -fold) of the antineoplastic activity of carboplatin in various cell lines, including malignant gliomas (Cohen et al., 1989, 1990). In this regard, thymidine-carboplatin interaction increases with longer thymidine exposures up to $16 \mathrm{~h}$ and then plateaus. For doses of thymidine between 100 and $1000 \mu \mathrm{g} / \mathrm{ml}$ or 60 and $240 \mathrm{~g}$ thymidine $/ \mathrm{m}^{2}$, this sensitization effect is constant (Cohen et al., 1989, 1990).

In our prior phase I study (Robins et al., 1999), evaluating an infusion of thymidine alone $\left(75 \mathrm{~g} / \mathrm{m}^{2}\right.$ per $\left.24 \mathrm{~h}\right)$ or with escalating carboplatin (from 200 to $570 \mathrm{mg} / \mathrm{m}^{2}$ i.v. at hour 20), the thymidine pharmacokinetics observed were consistent with the literature. Significantly, thymidine did not affect the pharmacokinetics of carboplatin. Several patients, including 6 with recurrent high-grade malignant glioma, were treated, and the maximum tolerated dose was $480 \mathrm{mg} / \mathrm{m}^{2}$. Two of these glioma patients entered remission (1 partial; 1 complete); 1 patient had stable disease ( 7 months).

A corollary finding in this phase I study related to the observation that thymidine protected against carboplatin myelotoxicity (Robins et al., 1999). This clinical finding was predicted by preclinical modeling (Cohen and Robins, 1990). Several possible mechanisms could explain this observation. Thymidine is widely used to synchronize cells in S phase, with little cell death. Thymidine-induced synchronization also occurs in vivo for human hematopoietic progenitor cells (Blumenreich et al., 1984; Fox, 1989; Kufe et al., 1980a, 1980b). Because carboplatin has little cell cycle specificity, such synchronization would not enhance its myelotoxicity. However, the cessation of replication of progenitor hematopoietic cells by synchronization might allow additional time for repair of carboplatin-DNA adducts, resulting in relative myeloprotection.

Based on the aforementioned preclinical data and phase I study (Robins et al., 1999), as well as earlier studies demonstrating modest activity for carboplatin in highgrade gliomas (Poisson et al., 1991; Prados et al., 1996; Warnick et al., 1994; Yung et al., 1991), a phase II recurrent high-grade glioma study was initiated in July 1998 (Robins et al., 2000). This report summarizes the results of the phase II trial.

\section{Patients and Methods}

\section{Eligibility}

To be eligible for this protocol, patients were required to have histologically proven (with central pathology review) supratentorial malignant primary gliomas. These included $\mathrm{GBM}^{3}$ anaplastic astrocytoma, anaplastic oligodendroglioma, anaplastic mixed oligoastrocytoma, or malignant astrocytoma (not otherwise specified). Patients had to show unequivocal evidence for tumor recurrence or progression by MRI or CT scan performed within 21 days prior to registration. The prestudy scan was required to have $\geq 25 \%$ increase in the bidimensional size of the tumor. Patients with only evaluable disease (e.g., unidimensionally measurable lesions, or masses with margins not clearly defined) were not eligible for study. Those who had undergone recent resection for progressive tumor were eligible as long as there was residual evaluable disease that was documented by appropriate neuro-imagining, and they must have recovered from the effects of surgery. Postoperative scans were done either within 48 to $72 \mathrm{~h}$ of surgery or at least 3 weeks postoperatively. Patients were required to have failed prior radiation therapy and they were allowed no more than 2 prior chemotherapy regimens. They could not have received prior thymidine or platinum agents. All patients signed an informed consent (approved by the Institutional Review Board) indicating that they were aware of the investigational nature of this study. Patients had to be $\geq 16$ years old and have a life expectancy $>8$ weeks and were required to have a Karnofsky performance status of $\geq 60$. Patients must have recovered from the side effects of prior therapy and/or be at least 2 weeks from vincristine, 6 weeks from nitrosoureas, 3 weeks from procarbazine and 0 weeks from noncytotoxic agent administration (for example, interferon, tamoxifen, etc.). They were required to have adequate bone marrow function (white blood cell count $\geq 3000 / \mu 1$, absolute neutrophil count $\geq 2000 / \mathrm{mm}^{3}$, platelet count of $\geq 120,000 / \mathrm{mm}^{3}$, and hemoglobin $\geq 10 \mathrm{~g} \%$ ), liver function (serum glutamic oxaloacetic transaminase [SGOT] and bilirubin $<2$ times the upper limit of normal), and renal function (creatinine $<1.5 \mathrm{mg} / \mathrm{dl}$ and creatinine clearance $\geq 60 \mathrm{cc} / \mathrm{min}$ ) within 14 days before registration. Eligibility level for hemoglobin could be reached by transfusion. Patients were not on steroids or were on a stable dose for at least 7 days before registration. A stable dose of steroids was defined $<50 \%$ change. All patients were required to have a prestudy contrast MRI or CT 
scan of the brain within 21 days before registration on a stable steroid dose. If steroids were instituted or the dose changed since the scan was done, the scan had to be repeated before registration. The same types of scansMRI or CT - were used throughout the period of protocol treatment for tumor measurement. Patients with significant cardiac risk factors or gastrointestinal risk factors, such as active ulcerative colitis in the past 6 months, were ineligible. Patients with a history of any other cancer (except nonmelanoma skin cancer or carcinoma in situ of the cervix), unless in complete remission and off all therapy for that disease for a minimum of 3 years, were ineligible. Other ineligibility parameters included active infection, pregnancy, disease that would obscure toxicity or dangerously alter drug metabolism, or serious intercurrent medical illness.

A complete history and physical/neurological examination, as well as documentation of evaluable disease, was performed on all patients.

\section{Treatment}

Starting dosage and schedule. Thymidine was given $75 \mathrm{~g} / \mathrm{m}^{2}$ i.v. over $24 \mathrm{~h}$. (as a fixed dose) and carboplatin at an AUC of $7 \mathrm{mg} / \mathrm{ml} \times \mathrm{min}$. Dose calculations were made with the Bristol Laboratories Calvert formula AUC dose calculator (based on the creatinine clearance) (Princeton, N.J.). Carboplatin/thymidine cycles were repeated every 4 weeks.

Follow-up. Tumor response assessment was conducted after every other cycle, then during follow-up at least every 3 months until progression. Those with stable, partial or complete responses at the time of reassessment were treated with 2 additional cycles of combined thymidine-carboplatin therapy. Patients could remain on the study as long as there was no prohibitive toxicity, and as long as it was deemed that the therapy was clinically appropriate. If progressive disease was identified, the patients were removed from study. Patients with progressive disease were followed for survival whenever possible. A maximum of 6 cycles of therapy was permitted.

Toxicity monitoring included hematologic, hepatic, and renal function, electrolyte imbalance and any other greater than grade 3 toxicity. Although granulocyte colony-stimulating factor was allowed during the first cycle of therapy, it was not used for any patient. The Common Toxicity Criteria (National Institute of Health, version 2.0: http://CtEP.info.NIH.gov)was used for toxicity grading.

Thymidine infusion. Thymidine provided by the National Cancer Institute (CTEP) was prepared at a concentration of $30 \mathrm{mg} / \mathrm{ml}$ in a solution of $0.6 \% \mathrm{NaCl}$ solution to provide $75 \mathrm{~g}$ thymidine $/ \mathrm{m}^{2}$ over $24 \mathrm{~h}$ (4.25 liters over $24 \mathrm{~h}$ or $177 \mathrm{ml} / \mathrm{h}$ in a $1.7 \mathrm{~m}^{2}$ person).

Carboplatin infusion. Carboplatin was reconstituted with sterile water for injection and diluted with 5\% dextrose injection given as a 20 -min intravenous infusion without interrupting the thymidine infusion. Carboplatin and thymidine were administered through separate venous access sites. The carboplatin injection was begun after $20 \pm 0.5 \mathrm{~h}$ of thymidine infusion. The carboplatin dose was based on an AUC of $7 \mathrm{mg} / \mathrm{ml} \times$ min. Patients received antiemetic medications and other therapies according to standard practice guidelines. Supplementary magnesium sulfate was given during the thymidine infusion via a separate intravenous catheter, if indicated (i.e., by low serum levels). The initial carboplatin dose level was chosen because of the potential carboplatin dosemodifying effect of thymidine and the potential for some degree of thymidine myelosuppression in addition to the toxicity of carboplatin.

Carboplatin dose modifications. The carboplatin dose was escalated by 1 AUC unit in patients for subsequent cycles based on counts (i.e., AUC was increased by 1 AUC [e.g., 7 to 8], if there was less than grade 2 hematologic toxicity). The maximum AUC allowed was 10 .

Patients were not retreated unless there was recovery of platelets to $>100,000 / \mu \mathrm{l}$ and absolute neutrophil count of $>2,000 / \mu 1$, and resolution of any grade 2 or 3 nonhematologic toxicity. Treatment was held until lab values were stable and then dose reduction was instituted. Dose reduction was carried out by 1 AUC unit for the subsequent cycle of chemotherapy. If a patient required a dose reduction below AUC 4 based on toxicity, he/she was removed from protocol treatment.

The parameters for dose reduction by an AUC unit of 1 were as follows: nadir absolute neutrophil count $<500 / \mu \mathrm{l}$ for $>3$ days, and/or nadir platelets $<25,000 / \mu 1$. If successive cycles resulted in similar levels of myelosuppression, subsequent cycles were reduced an additional 1 AUC unit. Platelet transfusions were encouraged for

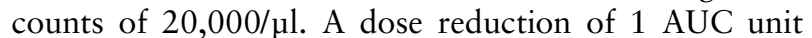
was instituted for febrile neutropenia. A dose reduction of 1 AUC unit took place for a dose delay (due to toxicity) of $>21$ days between cycles. Subsequent cycles beyond 21 days required further dose reductions of 1 AUC unit.

\section{Criteria for Evaluation and End Point Definitions}

All lesions were measured bidimensionally at each evaluation.

Complete response was defined as complete disappearance of all measurable and evaluable disease with no new lesions. Partial response was defined as greater than or equal to $50 \%$ decrease in the sum of products of perpendicular diameters of all measurable lesions compared with the baseline study. All responders had to be on stable or decreasing doses of dexamethasone. A less than $50 \%$ decrease was considered a minor response if it was sustained for at least 12 weeks. Stable disease was defined as no change for at least 12 weeks. Progression was scored with $\geq 25 \%$ increase in tumor bidimensional product, appearance of new lesions, or failure to return for follow-up because of death or deteriorating condition (unless clearly unrelated to the cancer). All responses were verified centrally by radiographic review.

Time to treatment failure was scored from the date of registration to the date of first observation of progressive disease, nonreversible neurologic progression, or permanently increased steroid requirement (applied to stable 
Table 1. Patient demographics

$\begin{array}{ll}\text { No. of patients entered } & 45 \\ \text { No. unevaluable } & 4(8.9 \%) \\ \text { Median age (range) } & 47(20-70) \\ \text { Sex } & \text { Female } 46 \% \text {; male } 54 \% \\ \text { Prior treatment } & \text { Surgery, } 90 \% \text {; external radiation } \\ & \text { therapy, 100\%; chemotherapy, } \\ & 81 \% ; \text { immunotherapy, } 7 \% \\ \text { Prior chemotherapy } & \text { None, 19.5\%; } 1 \text { regimen, } 61 \% ; \\ & 2 \text { regimens, 19.5\% } \\ \text { Histology } & \text { GBM, 76\%; AA, 10\%; AO, 12\%; } \\ & \text { mixed, } 2 \%\end{array}$

Abbreviations: $\mathrm{AA}$, anaplastic astrocytoma; $\mathrm{AO}$, anaplastic oligodendroglioma.

disease only), death due to any cause, or early discontinuation of treatment. Time to death was scored from date of resignation to date of death due to any cause.

\section{Statistical Considerations}

The planned sample size was 40 patients (allowing for $10 \%$ ineligibility).

The primary endpoint of the phase II study was response. A true response rate to carboplatin/thymidine of $20 \%$ or more would be of considerable interest, whereas further testing would not be planned if the true response rate was $6 \%$ or lower. Initially, 20 patients were accrued. If none of the first 20 had responded to treatment, we would have closed the study and concluded that the agent was inactive in this patient population. Five or more responses from a total of 40 patients was to be considered evidence warranting further study, provided other factors (such as toxicity, time to treatment failure, and survival) also appeared favorable.

The design had a significance level (probability of falsely declaring an agent with a $5 \%$ response probability to warrant further study) of .05 and power (probability of correctly declaring an agent with a $20 \%$ response probability to warrant further study) of $92 \%$. The chances of ending accrual after only the first stage, as well as the chances of concluding the agent warrants further study, over various true response probabilities are as follows:

True response probability: $.05, .10, .15, .20$
Probability of early stopping: 36\%, 12\%, 4\%, 1.\%

Probability of declaring agent warrants further study $5 \%, 37 \%, 73 \%, 92 \%$

The accrual of 40 patients to the phase II portion of this study was considered sufficient to estimate the true response rate, toxicity rate, or 4-month treatment failure rate to within, at most, $+16 \%$. Any toxicity occurring with at least a $5 \%$ probability would likely be seen at least once (with an $87 \%$ chance).

\section{Results}

Table 1 summarizes the demographics of our patient population. There was an almost equal male-to-female distribution; median age was 47. Most importantly, the high number of prior therapies indicated that this was a poor risk population; most had GBM with prior resection, radiation, and chemotherapy. Four patients were unevaluable: 1 withdrew from study before treatment; 2 had improper baseline MRIs; 1 had inadequate pretreatment hematologic parameters.

As observed in our earlier clinical trial (Robins et al., 1999), carboplatin-induced thrombocytopenia was dose limiting (Table 2). However, as was also the case in the earlier trial, there was an apparent myeloprotective effect of thymidine allowing for dose escalation of carboplatin up to an AUC of 10 (Table 2). In order to evaluate this myeloprotection, we applied modeling developed by Egorin's Group (Jordell et al., 1992), which predicts carboplatin hematologic toxicity based on prior therapy. Tables 3 and 4 compare the actual toxicity to the predicted outcomes for white blood cell and platelet toxicity, respectively.

Table 5 summarizes the nonhematologic toxicities encountered. These were comparable to our earlier phase I study. Nausea and vomiting were responsive to antiemetics; prophylactic antiemetics were effective. Headaches were responsive to analgesics. Thymidinerelated toxicity generally occurred midway through infusion. Overall, this regimen was well tolerated.

\section{Disease Response to Therapy}

We observed 2 patients with partial remission $(4.8 \%$; 1 anaplastic oligodendroglioma, 1 GBM), 3 with minor

Table 2. Toxicity with carboplatin and thymidine therapy

\begin{tabular}{|c|c|c|c|c|}
\hline \multirow[b]{2}{*}{ CBDCA-AUC ${ }^{a}$} & \multirow[b]{2}{*}{ No. of courses } & \multicolumn{2}{|c|}{ Median nadir cells/ $\mu \mathrm{l}$ [day] (range) } & \multirow[b]{2}{*}{ No. evaluable pts/SPR } \\
\hline & & Platelet (×1000) & ANC $(\times 100)$ & \\
\hline 5 & 3 & $70[15](51-77)$ & $1.7[28](1.4-1.7)$ & $1 / 1$ \\
\hline 6 & 4 & $70[21.5](50-137)$ & $2.5[12](1.3-3.3)$ & $5 / 3$ \\
\hline $7^{b}$ & 58 & 110 [22] (10-314) & $2.3[19](.5-6.9)$ & $41 / 20$ \\
\hline 8 & 20 & $133[16.5](34-178)$ & $2.5[22](.4-10.9)$ & $14 / 6$ \\
\hline 9 & 11 & $112[15](35-205)$ & $2.5[22](1.1-5.5)$ & $7 / 3$ \\
\hline 10 & 5 & $114[4.5](54-164)$ & $1.6[9](1.2-2.5)$ & $3 / 3$ \\
\hline
\end{tabular}

Abbreviations: ANC, absolute neutrophil count; AUC, area under the curve; pts, patients; CBDCA, carboplatin; SPR, number of patients exhibiting white blood cell count <1.5 and/or platelet $<100$.

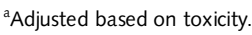

${ }^{\mathrm{b}}$ Starting AUC. 
Table 3. Incidence of white blood cell toxicity greater than or equal to grade 3

\begin{tabular}{lcccc} 
& \multicolumn{4}{c}{ Predicted $^{\mathrm{a}}(\%)$} \\
\cline { 2 - 4 } If prior chemotherapy & If no prior chemotherapy & Estimated for study $($ CBDCA/TdR) & $>24$ & Actual (\%) \\
\hline 6 & 30 & $<5$ & 29 & 0 \\
7 & 35 & 5 & 34 & 5 \\
8 & 40 & 10 & 46 & 9 \\
9 & 50 & 30 & 53 & 0 \\
10 & 55 & 45 & & 5 \\
\hline
\end{tabular}

Abbreviations: AUC, area under the curve; CBDCA, carboplatin; $T d R$, thymidine.

aBased on modeling (Jordell et al., 1992).

Table 4. Incidence of platelet toxicity greater than or equal to grade 3

\begin{tabular}{lcccc} 
& \multicolumn{4}{c}{ Predicted $^{\mathrm{a}(\%)}$} \\
\cline { 2 - 4 } AUC & If prior chemotherapy & If no prior chemotherapy & Estimated for study (CBDCA/TdR) & Actual (\%) \\
\hline 6 & 30 & 10 & 26 & 0 \\
7 & 45 & 20 & 40 & 15 \\
8 & 50 & 30 & 56 & 10 \\
9 & 55 & 50 & 64 & 9 \\
10 & 60 & 60 & 60 & 20 \\
\hline
\end{tabular}

Abbreviations: see Table 3.

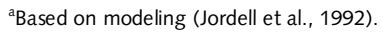

Table 5. Incidence of nonhematologic toxicity with carboplatin and thymidine therapy

\begin{tabular}{lcccc} 
Grade & Headache (\%) & Altered consciousness (\%) & Fatigue (\%) & Nausea (\%) \\
\hline 1 & 5 & 6 & 13 & 2 \\
2 & 8 & 1 & 1 & 7 \\
3 & 4 & 1 & 0 & 0 \\
4 & 0 & 2 & 0 & 0 \\
\hline
\end{tabular}

responses $(7.3 \% ; 1$ anaplastic astrocytoma, 2 GBMs), 6 with stable disease $(14.6 \%)$ and 30 with progressive disease $(73.2 \%)$. Kaplan-Meier curve estimates of survival and time to progression were calculated for the GBM patients. The median survival was 23 weeks with a $95 \%$ CI of 20 to 50 weeks. At weeks 13, 26, 39, 52, and 78 , the Kaplan-Meier values for median survival (95\% CI) were, respectively, 0.87 (0.76, 1.00), 0.45 (0.31, 0.67), 0.39 (0.25, 0.60), 0.23 (0.12, 0.43$)$, and $0.10(0.03,0.28)$. The median time to progression was 8 weeks with a $95 \%$ CI of 7 to 16 weeks; the KaplanMeier values for median progression-free survival $195 \%$ CI) for weeks 13,26 , and 39 were, respectively, 0.29 $(0.17,0.50), 0.13(0.05,0.32)$, and $0.03(.00,0.22)$. One of these patients (GBM) with a minor response continues with no evidence of disease progression at $21+$ months. Three patients are still alive with a median follow-up of 24 months.

\section{Discussion}

A significant clinical finding in this study relates to the reaffirmation that thymidine, given as a 24-h infusion, can provide an apparent effect in protecting against carboplatin-induced myelosuppression (Tables 3 and 4). A mechanistic explanation for this phenomenon may relate to synchronization of hematopoietic progenitor cells, a finding previously observed and described (Blumenreich et al., 1984; Fox, 1989; Kufe et al., 1980a, 1980 b). Such synchronization by thymidine may result in cessation of replication of hematopoietic cells, allowing for extended time for the repair of carboplatin-DNA adducts, and may result in relative myeloprotection. Alternatively, inhibition of poly(ADP-ribose) polymerase may preserve NAD cellular pools (NAD being the rate-limiting substrate for this enzyme). NAD preservation may protect progenitor hematopoietic cells from energy losses and/or apoptosis. This latter speculation is supported by studies of NAD levels in patients receiving either carboplatin alone, thymidine alone, or the combination (Robins et al., 1999).

It is interesting to note that an earlier clinical trial involving 1,3-bis(2-chloroethyl)-1-nitrosourea and thymidine provides putative evidence for thymidine protection against 1,3-bis(2-chloroethyl)-1-nitrosourea myelotoxicity (Schultz et al., 1996).

Most of the patients enrolled in this study had GBM (Table 1). For these patients, overall median survival and progression-free survival were comparable to other phase II experiences with GBM (Wong et al., 1999). Indeed, we are aware of no study to date involving recur- 
rent GBM patients that has reported an improvement in the $95 \%$ CI for time to recurrence (8-10 weeks) and overall survival (21-28 weeks) reported by Wong et al (1999) in their retrospective review.

Our response results are also consistent with earlier phase II carboplatin glioma trials. Prados et al. (1996), in their study of escalating doses of carboplatin, also review the literature for this drug as a single agent in recurrent high-grade gliomas. They point out that doses ranging from $350 \mathrm{mg} / \mathrm{m}^{2}$ to $>450 \mathrm{mg} / \mathrm{m}^{2}$ demonstrated no apparent difference in response rates (range, 7\%-14\%). The average response rate across studies by Poisson et al., 1991; Prados et al., 1996; Warnick et al., 1994; and Yung et al., 1991, involving 142 patients, was $\sim 10 \%$. We agree with their summary, as well as their observation that GBM patients were less likely to respond and had a shorter time to tumor progression. Parenthetically, the myelosuppression seen in these 4 studies was considerably higher than that seen with carboplatin/thymidine, consistent with data projected in Tables 3 and 4 .
Clearly the combination of thymidine and carboplatin provides no therapeutic advance in the treatment of GBM. Taken collectively, however, the results of this trial may provide an important foundation for future clinical trials. In this regard, temozolomide currently represents a promising new chemotherapeutic agent in the treatment of both glioma and malignant melanoma. Poly(ADPribose) polymerase represents a known resistance mechanism for this compound (Raymond et al., 1997; Tentori et al., 1997; Wedge et al., 1996). Thus, the combination of thymidine and temozolomide, as well as the addition of carboplatin and/or 1,3-bis(2-chloroethyl)-1-nitrosourea, represents an attractive multimodality approach to these diseases for which therapeutic gains have been exceedingly modest. The recognition of the myeloprotective value of thymidine is consistent with the practicality of such a multiagent approach. Preclinical studies now in progress are testing these combination therapies as a prelude to formal clinical multi-institutional trials.

\section{References}

Blumenreich, M.S., Woodcock, T.M., Andreeff, M., Hidemann, W., Chou, T.C., Vale, K., O'Hehir, M., Clarkson, B.D., and Yound, C.W. (1984) Effect of very high-dose thymidine infusions on leukemia and lymphoma patients. Cancer Res. 44, 2203-2207.

Cohen, J.D., and Robins, H.I. (1990) Thymidine enhancement of carboplatin cytotoxicity: In vivo studies in normal B6D2F1 mice. Cancer Lett. 55, 39-44.

Cohen, J.D., Robins, H.I., Schmitt, C.L., and Tanner, M.A. (1989) Interactions of thymidine, hyperthermia, and cis-diammine-1,1-cyclobutane dicarboxylate platinum (II) in human T cell leukemia. Cancer Res. 49, 5805-5809.

Cohen, J.D., Robins, H.I., and Javid, M.J. (1990) Sensitization of C6 glioma to carboplatin cytotoxicity by hyperthermia and thymidine. J. Neurooncol. 9, 1-8.

Fox, R.M. (1989) Changes in deoxynucleoside triphosphate pools induced by inhibitors and modulators of ribonucleotide reductase. In: Cory, J.G., and Cory, A.H. (Eds.), Inhibitors of Ribonucleoside Diphosphate Reductase Activity. New York: Pergamon Press. pp. 113-126.

Jordell, D.I., Egorin, M.J., Canetta, R.M., Langenberg, P., Goldbloom, E.P., Burroughs, J.N., Goodlow, J.L., Tan, S., and Wiltshaw, E. (1992) Relationships between carboplatin exposure and tumor response and toxicity in patients with ovarian cancer. J. Clin. Oncol. 10, 520-528.

Kornblith, P.L., and Walker, M. (1988) Chemotherapy for malignant gliomas. J. Neurosurg. 68, 1-17.

Kufe, D.W., Beardsley, P., Karp, D., Parker, L., Rosowsky, A., Canellos, G., and Frei, E., III. (1980a) High-dose thymidine infusions in patients with leukemia and lymphoma. Blood 55, 580-589.

Kufe, D.W., Egan, E.M., Rosowsky, A., Ensminger, W., and Frei, E., III. (1980b) Thymidine arrest and synchrony of cellular growth in vivo. Cancer Treat. Rep. 64, 1307-1317.

O'Dwyer, P.J., King, S.A., Hoth, D.F., and Leyland-Jones, B. (1987) Role of thymidine in biomedical modulation: A review. Cancer Res. 47, 3911-3919.

Poisson, M., Pereon, Y., Chiras, J., and Delattre, J.Y. (1991) Treatment of recurrent malignant supratentorial gliomas with carboplatin (CBDCA). J. Neurooncol. 10, 139-144.

Prados, M.D., Warnick, R.E., Mack, E.E., Chandler, K.L., Rabbitt, J., Page, M., and Malec, M. (1996) Intravenous carboplatin for recurrent gliomas: A dose escalating phase II trial. Am. J. Clin. Oncol. 19, 609-612.

Raymond, E., Izbicka, E., Soda, H., Gerson, S.L., Dugan, M., and Von Hoff,
D.D. (1997) Activity of temozolamide against human tumor colony-forming units. Clin. Cancer Res. 3, 1769-1774.

Robins, H.I., Tutsch, K., Katschinski, D.M., Mehta, M., Jacobson, E., Olsen, M., Cohen, J.D., Tiggelaar, C.L., Arzoomanian, R.Z., Alberti, D., Feierabend, C., and Wilding, G. (1999) A Phase I trial of intravenous thymidine and carboplatin in patients with advanced cancer. J. Clin. Oncol. 17, 2922-2931.

Robins, H.I., Prados, M.D., Rao, D.R., Chang, S., Yung, A., Hess, K., Schiff, D., Greenberg, H., Fink, K., Nicolas, K., Kuhn, J., and Mehta, M. (2000) A phase II trial of thymidine and carboplatin for recurrent malignant glioma: A North American Brain Tumor Consortium (NABTC) study. In: ASCO (Ed.), Thirty-sixth Annual Meeting of the American Society of Clinical Oncology, May 20-24, 2000, New Orleans, Louisiana: Program/Proceedings, Vol. 19. Alexandra, Va.: ASCO p. 166a. (abstract)

Schultz, M.Z., Sandler, A.B., Durivage, H.J., and Cooper, D.L. (1996) A phase I study of BCNU plus thymidine in patients with refractory cancer. Cancer Invest. 14, 218-224.

Tentori, L., Orlando, L., Lacal, P.M., Benincasa, E., Faraoni, I., Bonmassar, E., D'Atri, S., and Graziani, G. (1997) Inhibition of $O^{6}$-alkylguanine DNAalkyltransferase or poly(ADP-ribose) polymerase increases susceptibility of leukemic cells to apoptosis induced by temozolomide. Mol. Pharmacol. 52, 249-258.

Warnick, R.E., Prados, M.D., Mack, E.E., Chandler, K.L., Doz, F., Rabbitt, J.E., and Malec, M.K. (1994) A phase II study of intravenous carboplatin for the treatment of recurrent gliomas. J. Neurooncol. 19, 69-74.

Wedge, S.R., Porteous, J.K., and Newlands, E.S. (1996) 3-aminobenzamide and/or $\mathrm{O}^{6}$-benzylguanine evaluated as an adjuvant to temozolomide or $\mathrm{BCNU}$ treatment in cell lines of variable mismatch repair status and $\mathrm{O}^{6}$ alkylguanine-DNA alkyltransferase activity. Br. J. Cancer 74, 1030-1036.

Wong, E.T., Hess, K.R., Gleason, M.J., Jaeckle, K.A., Kyritsis, M.D., Prados, M.D., Levin, V.A., and Yung, W.K.A. (1999) Outcomes and prognostic factors in recurrent glioma patients enrolled onto phase II clinical trials. J. Clin. Oncol. 17, 2572-2578.

Yung, W.K.A., Mechtler, L., and Gleason, M.J. (1991) Intravenous carboplatin for recurrent malignant glioma: A phase II study. J. Clin. Oncol. 9, 860-864. 